\title{
Developments of EHM Under the Conditions of Big Data Technology
}

\author{
Jin Cai ${ }^{1, \text { a }}$, Hui Liu ${ }^{2, \text { b }}$, Zheng Wang ${ }^{3, \mathrm{c}}$ and Jihong Zhu ${ }^{2, \mathrm{~d}}$ \\ ${ }^{1}$ Faculty of Aerospace engineering, Shenyang Aerospace University, Shenyang, 110136, China; \\ ${ }^{2}$ School of Mechanical Engineering, Northwestern Polytechnical University, Xi'an, 710072, China; \\ 3 Technical Center, AECC Shenyang Liming Aeroengine Co.,Ltd. Shenyang,110043, China. \\ a saucai@163.com, bhuilxy@sohu.com, cwhitemark2001@163.com, dJjh.zhu@nwpu.edu.cn
}

\begin{abstract}
This paper explores the role of big data technology development in the promotion of aero engine health management (EHM) technology. Firstly, the research and development history of EHM is elaborated in detail, and then the key technologies for EHM research are analyzed. Finally, according to the existing difficulties and technical problems, the method of combining computer science engineering and aerospace science and technology is proposed to improve Chinese aviation EHM technology.
\end{abstract}

Keywords: big data, EHM, computer science engineering, aerospace science.

\section{Introduction}

The structure of modern aero-engine is complex, the working condition of the aero-engine is harsh and changeable, at the same time the aero-engine is an accident-prone system. The traditional way of regular maintenance not only consumes a lot of resources and has low efficiency, but also needs considerable costs. For example, the United States Department of Defense spends about \$1.3 billion annually on engine purchases, while the cost of maintaining existing engines is around $\$ 3.5$ billion. Therefore, in the defense procurement reforms conducted by the United States Department of Defense regard "economic affordability" as the primary driver in defense procurement reforms since the 1990s. Owing to its small size, high efficiency, economic affordability and avoidance of significant catastrophic accidents, the condition-based maintenance is very suitable for the maintenance of large complex systems. One of the prerequisites for the implementation of Condition Based Maintenance (CBM) is to require the system to get the ability to predict its own faults and manage its health status, which in turn leads to the concept of Prognostic and Health Management (PHM). PHM refers to the use of sensors to acquire the data information of the system, using various intelligent models and algorithms to evaluate the health status of the system itself, predicting before the system failure occurs, and providing a series of maintenance support measures in combination with the available resource information $[1,2]$. Realizing the system's conditional maintenance as the application of PHM technology in aero-engine, EHM is based on the above-mentioned background, based on the outdate engine condition monitoring and fault diagnosis, comprehensive utilization of information technology, artificial intelligence and so on [3]. One of the prerequisites for CBM is that the system has the ability to predict its own faults and manage health status. Therefore, the concept of PHM has been made to achieve "economic affordability" [4-6].

PHM refers to the use of sensors to acquire the data information of the system. With the help of layered intelligent inference engine software, using fewer sensors or even special sensors to predict, diagnose and isolate faults on the aircraft. The introduction of PHM is not to directly eliminate the fault, but to assess the health status of the system, to forecast before the system failed, and to provide a series of maintenance support measures in combination with the available resource information to achieve the system maintenance.

With the development of PHM, especially the concept of Industry 4.0 was put forward after 2010, PHM has been shifting the center from component/machine to user health management, in which big data analysis and application, health management based on artificial intelligence and cloud computing will play an important role in the PHM. In the era of big data, improving the research and service level of health management technology can provide regional service and life cycle service solutions 
for our aviation engines better, thus achieving the objective of safeguarding capacity of high digitization, networking, integration, real-time and intelligent. In the era of big data, more and more data collection and analysis tools will be added to the system of EHM, providing technical support for maintenance support personnel to determine the cause of failure and maintenance measures in a timely manner and to arrange maintenance time reasonably. So as to achieve predictive maintenance, improve the rate of the engine integrity, the rate of the aircraft dispatch again, to enhance the capability to fight.

\section{Development Process of EHM System Technology}

In terms of civil engines, foreign began to study the condition monitoring and fault diagnostics system for aero-engine in the 1960s. In the 1970s, the state monitoring system began to be applied in civil engines and achieved success, which improved flight safety and flight operation efficiency. In the 1980s and 1990s, the rapid development of electronic technology and computer technology promoted the development of state monitoring and fault diagnosis technology for aero-engines greatly. Engines on the Boeing 747/767 and the Airbus A310 are equipped with the condition monitoring and fault diagnostic systems. By the beginning of the 21 st century, countries such as Europe and the United States proposed and implemented the concept of EHM in the B787 and A380 projects, marking the new stage of CBM and safety, maintainability and economic monitoring of aero-engine. The EHM system used by GENX engine of the GE and the British Rolls-Royce Trent 900 engine is a characteristic of a modern engine system. The EHM system is include in the airborne part and the ground part. The airborne part installed on the engine firstly, and the web-based remote monitoring and diagnosis are realized by means of an aircraft communication addressing and reporting system. Mainstream development direction of EHM systems. At present, the EHM system in foreign has been drafted into the engineering verification stage. Numerous technologies have been demonstrated and validated, and the implementation of PHM can reduce the cost of engine use and maintenance significantly, and improve the reliability and safety of the engine.

In the military engine, the MK202 "Spay engine "developed by Rolls-Royce in the 1960s was provided with a lubricating oil monitoring system. In the 1970s, the T700-GE-700 and T700-GE 701 turboshaft engines developed by GE had the components and functions of the initial health monitoring system, which can perform basic state monitoring and complete fault diagnosis of important parts. The control system of the T700-GE-701C engine consists of Digital Electronic Controller (DEC) and Hydro Mechanical Unit (HMU), and is fitted with a history recorder. Its health monitoring function is primarily realized by DECU and history recorder. The IECMS, which GE designing for the F404-GE-400 turbofan in 1979, is a real-time engine monitoring and life tracking system. The United Kingdom developed the Engine Usage Monitoring System (EUMS) and the Low Cycle Fatigue Counter (LCFC) in 1975.

After the 1980s, with the development of engine and computer technology, engine monitoring systems have increasingly become a standard configuration. The UK combined the experience with both EUMS and LCFC, developed the unit diagnostic system of the popularity of cluster. In 1982, Pratt \& Whitney in the USA began to develop the state monitoring system of the F100-PW-200 engine. By 1987, the system was compatible with aircraft integration and logistics databases, and the system continued to expand and improve. In the late 1980s, the EJ200 engine jointly developed by four European countries proposed the progress requirements for state monitoring and fault diagnosis. The state monitoring and fault diagnosis functions was integrated in the FADEC system. With the development of technology, the state monitoring function of the EJ200 engine has been relatively perfect, reaching its progress requirements.

In the 1990s, the F119 engine of the US developed the Diagnostic and Health Management (DHM) system [8,9]. In addition to its comprehensive status monitoring function, the system also has a relatively complete fault diagnosis capability, but it has not yet achieved sufficient diagnostic accuracy and don't have clearly assessment indicators. Although the F119 engine DHM system does not have predictive capabilities yet, it is the most advanced intelligent state monitoring and fault 
diagnosis system for active aero engines. The F135 engine is a follow-up improvement of the F119 engine, in addition to its comprehensive condition monitoring and fault diagnosis capabilities, the requirements for fault prediction are proposed, and also have certain predictive capabilities. Prediction is one of the distinguishing features of the health management system that distinguishes it from the engine monitoring and diagnostic system of the early stage. To this end, the Pratt \& Whitney Company of the US invested a lot of resources, developed a new type of sensor and diagnostic software, and formed a relatively complete health management system and supporting assessment indicators system. Health management technology is fully applied in the development of the F135 engine, representing the highest level achievable in the United States currently.

In summary, the technical development of the EHM system has undergone the process shown in Figure 1. From the state monitoring, fault diagnosis to the gradual development and improvement process of forecasting and health management, the early stage engine health management system exists in the form of simple or basic state monitoring system and fault diagnosis system; then state monitoring and fault diagnosis technology development and maturity continuous, and form a

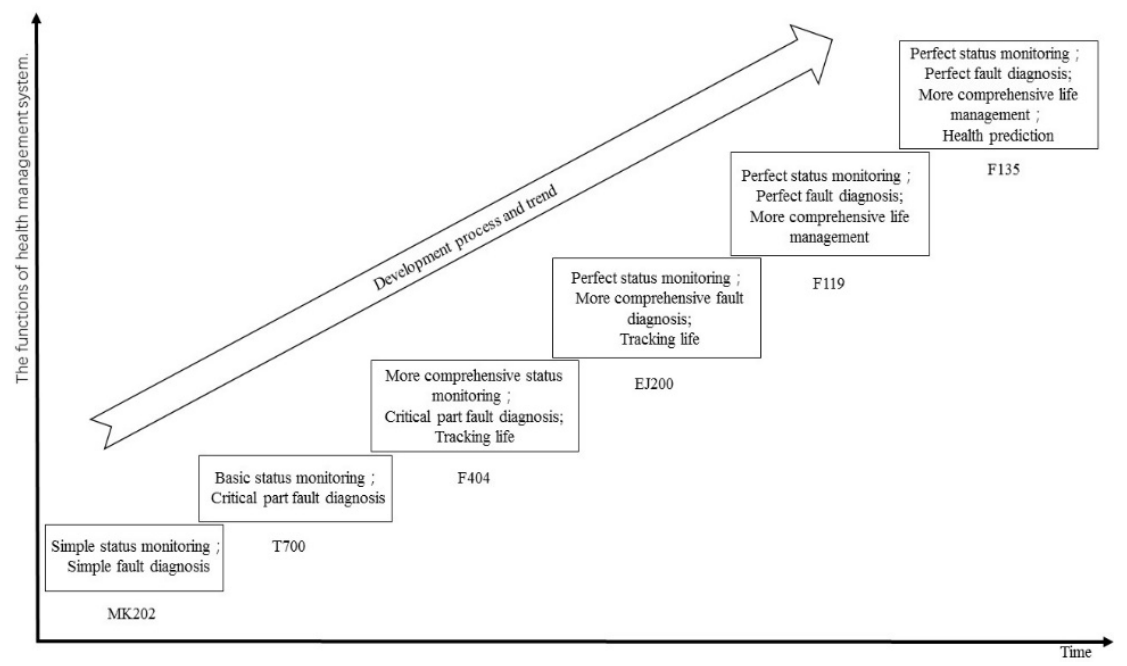

Fig. 1 The technical development of EHP

complete state monitoring and fault diagnosis system gradually; and then gradually combined with engine life management and forecasting technology, developed into the modern aviation EHM system.

\section{The Key Technologies of EHM}

EHM involves not only many disciplines related to the engine, but also many aspects of technology, such as sensors, data management, various modeling methods and algorithms, information fusion, prediction technology, system integration technology. Here we focus on the key technologies that distinguish EHM from traditional condition monitoring and trouble shooting.

\subsection{Data Management}

In the field of EHM, data mining technology has played an important role and attracted great attention. For example, NASA launched the Aviation Safety Program (AvSP) in 1999. The development goal aims to reduce the accident rate by $80 \%$ in 2007 and by $90 \%$ in 2025 . The plan details the development roadmap for aircraft engine health management data mining tools, and identifies research goals to achieve significant improvements in safety, reliable prediction of faults andthe remaining component life, and new laws to improve safety and reliability of data [10]. Data mining and advanced reasoning have also received great attention in the long-term development planning of VAATE, and as the main technology control way for reducing the maintenance work hours and increasing the unplanned change interval in the advanced health management system forecasting, diagnosis and maintenance process. 


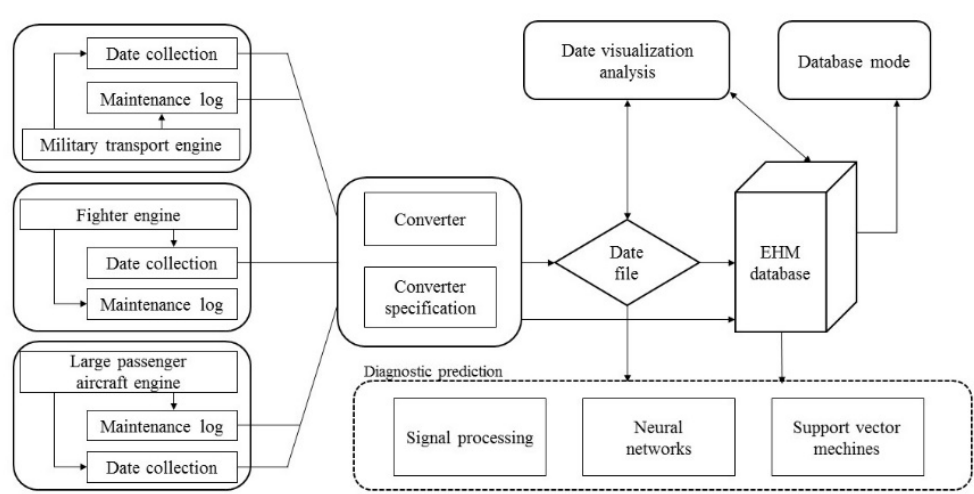

Fig. 2 The concept of data management for EHM

The EHM may be targeted as engines from different sectors and different types of commercial or military aircraft. The engines of fighter aircraft, large military transport aircraft and civil aviation trunk aircraft are typical representatives. The EHM system requirements and generated data types, a complex logical structure, large diagnostic and forecasting data requirements, and high requirements for real-time and security of data. In order to increase the versatility and efficiency of the EHM system, reduce costs and realize cross-platform EHM, it is necessary to manage engine data from different platforms. Figure 2 displays the data management scheme for the characteristics of EHM. Engine data from different platforms are converted to a uniform format by data converters in the same conversion specification and stored as standard data files and databases for use in a variety of processing procedures, including diagnostics, predictive algorithms, and visualization.

\subsection{Information Fusion}

Information fusion is a process of comprehensive processing of multi-source information at multiple levels, in which each level reflects the abstraction of different levels of original observation data [11].

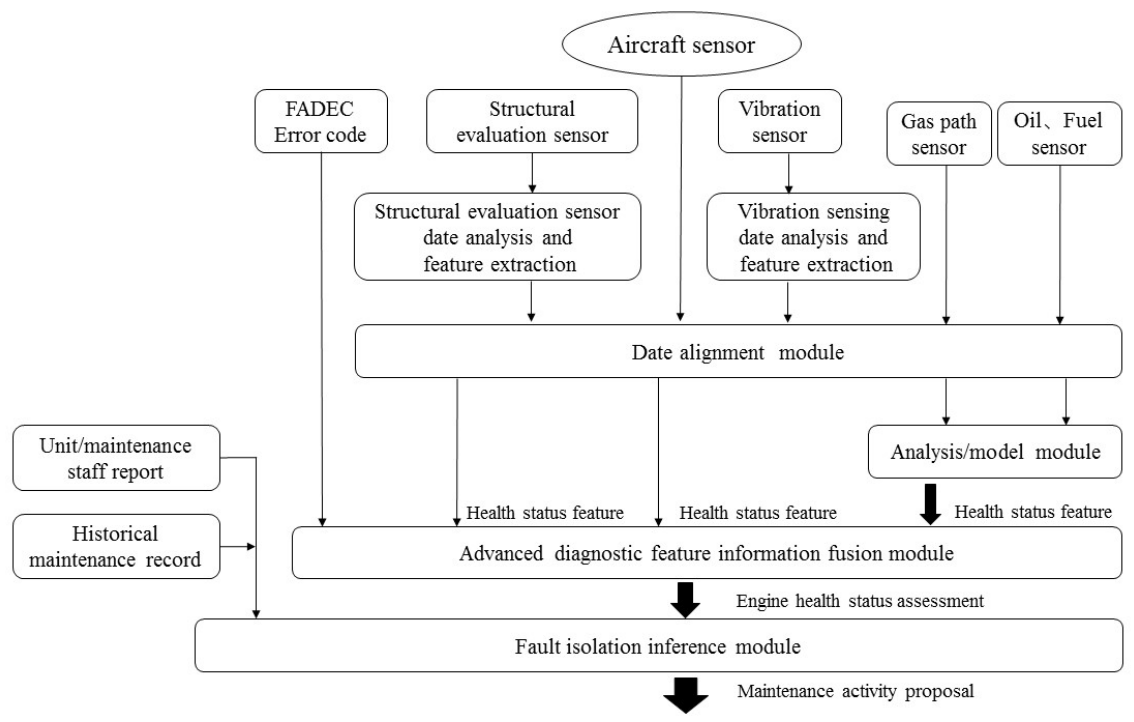

Fig.3 The architecture of information fusion for EHM

The purpose of information fusion in EHM is to improve the accuracy and credibility of engine fault diagnosis, performance prediction, and residual life estimation of critical components. Engine status information comes from a variety of data sources, including sensor system measurements, 
component design models, engine historical maintenance records, Full Authority Digital Engine Control (FADEC) system generated fault codes, crew, maintenance personnel reports and so on. From the perspective of information processing, the ultimate goal of EHM is to extract useful information from diverse data sources through information fusion to obtain comprehensive diagnosis and prediction knowledge about engine health status, and make the optimal decisions finally. The more mature EHM information fusion system was developed jointly by the Dryden Flight Research Center and Pratt \& Whitney for the F117 engine. The architecture is illustrated in Figure 3[12]. The system requires a component-based, open, hierarchical architecture that performs signal processing, data analysis, health assessment, fault diagnosis reasoning, maintenance activity recommendations and so on.

\subsection{Technology of Prediction}

The technology of prediction is the highest level of reasoning in EHM and is a further fusion of results such as fault detection, fault isolation and so on. In terms of EHM, the prediction mainly includes predicting the timing of component failure, calculating the remaining life of key components, and analyzing the trend of engine performance degradation. The biggest difference between prediction technology and condition monitoring and fault diagnosis is possible that the prediction emphasizes quantitative analysis. Prediction techniques can be divided into three categories essentially: $[13,14]$.

\section{(1)Prediction Based on the Model}

The precondition for using model-based prediction is that there must be an accurate engine mathematical model and the failure model of the component. The most prominent advantage of model-based prediction is the ability to meet real-time requirements, so model-based predictions are typically used on airborne EHM systems, such as the enhanced adaptive airborne real-time engine model used by the F117 engine of the C-17. Since the engine is a complicated nonlinear system, it is difficult to establish an accurate mathematical model, so the scope and effect of model-based prediction in practical applications are limited.

\section{(2)Prediction Based on the Knowledge}

The feature of knowledge-based prediction is that it does not require an accurate mathematical model and can make full use of the expert knowledge and experience in various disciplines of the engine. The two most typical forms of application for the knowledge-based prediction are expert systems and fuzzy logic. Fault diagnosis is a traditional application field of expert systems. In recent years, expert systems have also been introduced in predictions, and often combined with other technologies such as neural networks. However, due to the "bottleneck" problem of knowledge acquisition and knowledge representation in the expert system, it has a certain locality. Fuzzy logic is used as a tool for expressing and dealing with fuzzy concepts, with the ability to process uncertainty information. Fuzzy prediction can use expert knowledge to construct a fuzzy rule base, which can take full advantage of expert knowledge and experience, and a properly designed fuzzy logic system can approximate a given nonlinear function with arbitrary precision. Since fuzzy prediction is still in the research stage, some problems need to be addressed.

\section{(3)Prediction Based on the Data}

The biggest advantage of data-based prediction is that no precise mathematical or physical model of the engine is required, the most typical example is the neural network [15]. Neural network technology is commonly used in prediction, and often combined with other technologies to derive various forms of neural network structure. In addition to neural networks, there are additional databased prediction methods, such as Bayesian networks, Hidden Markov Models, and data mining.

For complex systems such as engines, due to the difficulty of prediction research, it is difficult to guarantee the application effect by using a single method for prediction. The hybrid prediction method can not only fully absorb the advantages of the respective methods, but also make up for their respective shortcomings. Therefore, it is an inevitable trend to predict the development of technology 
by combining various different prediction methods and further improving the comprehensive performance of prediction [16-18].

\subsection{Technology of System Integration}

\section{(1) Integration of the EHM System}

The EHM system is a complex information system that needs to be analyzed and developed using expert knowledge, techniques, and models from different disciplines. The EHM system consists of a variety of distinct modules, but the data flow is same roughly, such as signal source, signal conditioning, data processing, data storage, and data retrieval. Integration can improve the efficiency of EHM system development, achieve the sharing of capabilities of each module, and benefit the modules and systems of the EHM system. Integration can treat the EHM system as a whole, helping to balance the analysis system to meet different functional requirements, configurations, or architecture. Integration provides a set of options and solutions for EHM systems to meet diverse needs.

\section{(2) Integration of EHM System with Engine Control System}

The current advanced engines basically use the Full Authority Digital Electronic Control (FADEC) system. The relationship between the EHM system and the FADEC system is very similar. Most of the necessary parameters for the EHM system are also required for the FADEC system, such as the speed at high and low-pressure rotor, exhaust temperature, fuel flow, and total engine intake air temperature. Some features of the EHM system are also needed in the FADEC system, such as sensor fault shooting and overrun checking. In the EHM system, the overrun check is used to alert the crew and the ground crew, while the FADEC system changes the control law according to the report output by the EHM system for the engine health status to mitigate the effects of abnormal conditions to ensure engine safety. In view of the close relationship between the two, integration of the EHM system and the FADEC system is an important development trend for the new generation of engines.

\section{(3) Integration with Other Onboard Systems}

Other onboard systems are also linked to multiple links in the EHM system, such as cockpit display systems, flight management systems, mission management systems. The numeral system and data bus enable the EHM system to be integrated with other on-board systems to increase system development efficiency, reduce costs, and reduce space and weight.

\section{Suggestions on the Development of EHM}

1) Deepen the research of basic theory, grasp the mechanical performance data of fatigue, fracture, creep and corrosion in material properties, and relate with the advanced nature, reliability and safety of engine development closely. Describe the trend of failure over time based on environmental conditions, engine operation, and current state of the component;

2) Aiming at the difference in fatigue life of rotating parts of aero-engines, the surface strengthening of the parts is carried out by using the metal surface modification process, so that the metal grains are refined or even nanocrystallization, thereby improving the high-cycle fatigue life of the engine components and keeping the engine technical state consistent.

3) Accelerating the research of advanced hardware products not only needs to greatly improve the performance of hardware products, but also strengthens in terms of reliability, robustness, and harsh environmental adaptability. At the same time, various hardware resources should be optimized and scheduled so that they can be utilized fully and reasonably, and achieve the optimal overall performance of the entire hardware system;

4) At the engine design and development stage, EHM is valued as a design feature of the engine. The design idea is to examine how to obtain engine information more conveniently and accurately, and how to apply to the engine safely and smoothly. EHM as an integral part of the engine design, the engine is designed to improve the surveillance, diagnosis, predictability, and mitigation of the engine, thereby maximizing the benefits of EHM. 


\section{References}

[1]. Merecr C R, Simon D L, Hunter G W, et al. Fundamental technology development for gas-turbine engine health management. 2007, NASA-TM-2007-0022364.

[2]. He Houbo, Zhao Jianmin, Xu changan. Cost-benefit Model for PHM. Procedia Environmental Sciences. Vol.10 (2011), p.759-764.

[3]. E. Scanff, K.L. Feldman, S. Ghelam, et al. Life cycle cost impact of using prognostic health management (PHM) for helicopter avionics. Microelectronics Reliability. 47(2007), 1857-1864.

[4]. Alexandre Popov, Wolfgang Fink, Carolyn Mcgregor, et al. PHM for astronauts: Elaborating and refining the concept. Aerospace conference. 2016, p.1-9.

[5]. Yan Huilin, Xu Dongli, Yang Hu. Deep diagnostics and prognostics: An integrated hierarchical learning framework in PHM applications. Applied Soft Computing. (2018), p. 1-10.

[6]. J. Lee, F. Wu, W. Zhao, et al. Prognostics and health management design for rotary machinery systems-reviews. Methodology and Applications, Mech. Syst. Signal Process. Vol. 42 (2014) No.1-2, p. 314-334.

[7]. Fei Meng, Jien Yang, Hang Xu, et al. The Technical Research of PHM in Aeronautical Mechanical and Electronical System Based on IETM. Proceedings of the First Symposium on Aviation Maintenance and Management.Vol.1 (2014), p.133-139.

[8]. N. Md Nor, M.A. Hussain, C.R. Che Hassan, Fault diagnosis and classification framework using multi-scale classification based on kernel Fisher discriminant analysis for chemical process system, Applied Soft Computing. Vol. 61 (2017), p. 959-972.

[9]. A Popov, W Fink, A Hess. PHM for Astronauts- A New Application. 2013 Annual Conference of the Prognostics and Health Management Society Proceedings, New Orleans, 2013.

[10]. ZX. Yang, X B. Wang, J H. Zhong. Representational learning for fault diagnosis of wind turbine equipment: a multi-layered extreme learning machines approach. Energies. Vol. 9 (2016), p. 1-17.

[11]. Information on: www. aeronautics. Nasa. Gov/ programs- avsafe. Htm.

[12]. Volponi A J, Brotherton T, Luppold R. Development of an Information Fusion System for Engine Diagnostics and Health Management. 2004, AIAA 2004-6461.

[13]. Z Wen, Y Liu. Applications of Prognostics and Health Management in aviation industry. 2011 Prognostics and System Health Managment Confernece. 2011, p. 978-983.

[14]. Y Lei, F Jia, J Lin, et al. An intelligent fault diagnosis method using unsupervised feature learning towards mechanical big data. IEEE Transactions on Industrial Electronics. Vol. 63 (2016), p. 3137-3147.

[15]. A.M.D. Younus, B. S. Yang. Intelligent fault diagnosis of rotating machinery using infrared thermal image. Expert System with Applications. Vol.39 (2012), p. 2082-2091.

[16]. Jaw L C. Recent Advancements in Aircraft Engine Health Management (EHM) Technologies and Recommendations for the Next Step. 2005, ASME GT2005-68625.

[17]. Wen Zhu Sun, Jian Ling Qu, Yu Ping Zhou, et al. A Novel Estimation Method on Delta Engine Gas Temperature of a Certain Aero-Engine Based on Flight Data. Applied Mechanics and Materials. Vol. 203(2012), p. 375-379.

[18]. Melcher K J, Maul W A, Garg S. Propulsion Health Management System Development for Affordable and Reliable Operation of Space Exploration Systems. 2007, AIAA 2007-6237. 